\title{
Mycoplasma exploits mammalian tunneling nanotubes for cell-to-cell dissemination
}

\author{
Bong-Woo Kim ${ }^{1,2}$, Jae-Seon Lee $e^{3}$ Young-Gyu Ko ${ }^{1,4, *}$ \\ ${ }^{1}$ Tunneling Nanotube Research Center, Korea University, Seoul 02841, ²Skin Innovation R\&D Centre, HnB9 Co., Ltd. Cheongju 28161, \\ ${ }^{3}$ Department of Molecular Medicine, College of Medicine, Inha University, Incheon 22212, ${ }^{4}$ Division of Life Sciences, Korea University, \\ Seoul 02841, Korea
}

Using tunneling nanotubes (TNTs), various pathological molecules and viruses disseminate to adjacent cells intercellularly. Here, we show that the intracellular invasion of Mycoplasma hyorhinis induces the formation of actin- and tubulin-based TNTs in various mammalian cell lines. $M$. hyorhinis was found in TNTs generated by $M$. hyorhinis infection in NIH3T3 cells. Because mycoplasma-free recipient cells received mycoplasmas from $M$. hyorhinis-infected donor cells in a mixed co-culture system and not a spatially separated co-culture system, direct cell-to-cell contact via TNTs was necessary for the intracellular dissemination of $M$. hyorhinis. The activity of Rac1, which is a small GTP binding protein, was increased by the intracellular invasion of $M$. hyorhinis, and its pharmacological and genetic inhibition prevented $M$. hyorhinis infection-induced TNT generation in NIH3T3 cells. The pharmacological and genetic inhibition of Rac1 also reduced the cell-to-cell dissemination of $M$. hyorhinis. Based on these data, we conclude that intracellular invasion of $M$. hyorhinis induces the formation of TNTs, which are used for the cell-to-cell dissemination of M. hyorhinis. [BMB Reports 2019; 52(8): 490-495]

\section{INTRODUCTION}

Mammalian cells communicate with each other to control their proliferation, differentiation, survival and death (1). Intercellular communication occurs through secreted ligands (cytokines, chemokines, growth factors and hormones), extracellular vesicles (exosomes and ectosomes) and direct physical contacts (gap junctions and cellular synapses) (2, 3). Cell-to-cell communication also occurs via long, thin

*Corresponding author. Tel: +82-2-3290-3453; Fax: +82-2-9279028; E-mail: ygko@korea.ac.kr

https://doi.org/10.5483/BMBRep.2019.52.8.243

Received 15 October 2018, Revised 30 October 2018, Accepted 4 November 2018

Keywords: Fibroblast, Mycoplasma, Rac1, Tunneling nanotube membrane extensions called cytonemes in Drosophila and tunneling nanotubes (TNTs), membrane nanotubes (MNTs) and long-distance filopodia in vertebrate (4). TNTs have also been found open- or close-ended structures in rat pheochromocytoma PC12 cells, Raw264.7 cells and Jurkat T cells (5-7).

TNTs intercellularly transfer mitochondria as well as small vesicles from the endoplasmic reticulum, Golgi complexes, and the early endosomes (8). TNTs also transfer various pathological molecules, such as amyloid $\beta$ peptides, Tau, prion, and $\alpha$-synuclein, disseminating these molecules into the surrounding cells (9-11). Murine leukemia virus (MLV), influenza virus and herpes viruses also utilize TNTs to increase their dissemination efficiency (12-14). TNTs generated by human immunodeficiency virus (HIV) infection in macrophages are necessary for the efficient cell-to-cell dissemination of HIV viruses (15). In addition, human monocyte-derived macrophages utilize TNTs to trap and pull Mycobacterium bovis toward macrophage cell bodies (16).

Because mycoplasma without a cell wall is resistant to antibiotics, such as penicillin, which inhibits bacterial cell wall synthesis, mycoplasma often contaminates mammalian cell cultures. Mycoplasma infection changes cellular metabolism and growth, destroying mammalian cell lines (17). Several mycoplasma species are pathogenic in livestock and humans. Mycoplasma hyorhinis is a major cell culture contaminant that is often found in the swine respiratory tract and human skin (18). Mycoplasma adheres to the outside of the host cell membrane, invades host cells and survives in intracellular vesicles in the host cells, avoiding immunological surveillance (19). For example, Mycoplasma fermentans and Mycoplasma hominis are found in the vacuolar membrane of HeLa cells, which is the proliferation site of mycoplasma $(20,21)$.

In this present study, we demonstrate that $M$. hyorhinis infection induced the formation of TNTs in various mammalian cells by activating Rac1, which is a small GTP binding protein. Intracellular M. hyorhinis were transferred from infected cells to adjacent cells through these TNTs. The pharmaceutical or genetic inactivation of Rac1 inhibited $M$. hyorhinis-induced TNT formation and prevented the cell-to-cell dissemination of M. hyorhinis. Thus, we conclude

ISSN: 1976-670X (electronic edition)

Copyright (c) 2019 by the The Korean Society for Biochemistry and Molecular Biology

(c) This is an open-access article distributed under the terms of the Creative Commons Attribution Non-Commercial License (http://creativecommons.org/licenses/by-nc/4.0) which permits unrestricted non-commercial use, distribution, and reproduction in any medium, provided the original work is properly cited. 
that M. hyorhinis exploits TNTs for cell-to-cell dissemination.

\section{RESULTS}

\section{Mycoplasma hyorhinis infection induced TNT formation} Although various mammalian cells have been known to possess TNTs, TNTs were not observed in various cell lines, such as NIH3T murine fibroblasts, CCD986-sk human fibroblasts, Huh7 human hepatocarcinomas and B16F10 murine melanomas, by differential interference contrast (DIC) microscopy (Supplementary Fig. 1A, left panels). However, TNTs were observed in the extracellular space of these live cell lines after infection with Mycoplasma hyorhinis ( $M$. hyorhinis) for 7 days (Supplementary Fig. 1A, middle panels). After the M. hyorhinis infection, TNTs were found in most $\mathrm{NIH} 3 \mathrm{~T} 3$ cells, and each cell produced three or more TNTs; the other cell lines had results similar to those observed in NIH3T3 cells (Supplementary Fig. 1B). The TNTs disappeared from the $M$. hyorhinis-infected cell lines in the presence of mycoplasma removal agents (MRA) that specifically inhibit mycoplasma DNA gyrase (Supplementary Fig. 1). Thus, we conclude that $M$. hyorhinis infection induces TNT formation in various mammalian cell lines.

Subsequently, we determined the intracellular or extracellular location of mycoplasmas from M. hyorhinis-infected NIH3T3 cells by immunofluorescence staining of the P70 protein, which is a cell surface antigen of $M$. hyorhinis. In the presence of gentamicin, which kills only extracellular mycoplasmas without affecting intracellular mycoplasmas because of its limited penetration into mammalian cells (20), the P70 antigen disappeared from the non-permeabilized NIH3T3 cells but not from the permeabilized cells (Supplementary Fig. 2), indicating that $M$. hyorhinis adheres to the host cell surface and invades host cells. To determine whether extracellular or intracellular M. hyorhinis induces TNT formation in NIH3T3 cells, we observed TNTs in the gentamicin-treated cells. As shown in Fig. 1A, M. hyorhinis-induced TNT formation was not altered by the gentamicin treatment, whereas TNT formation was inhibited by MRA, which kills both extracellular and intracellular mycoplasmas (Fig. 1B). Thus, we conclude that intracellular M. hyorhinis induces TNT formation.

\section{M. hyorhinis is localized in TNTs}

To more clearly observe the TNTs in the extracellular space of the NIH3T3 cells after the $M$. hyorhinis infection, $M$. hyorhinis-free and -infected cells were stained with CellMast ${ }^{\mathrm{TM}}$, which is a specific tracker of the plasma membrane. TNTs were observed after the $M$. hyorhinis infection in the live state. However, the TNTs disappeared after the paraformaldehyde fixation and permeabilization induced by Triton X-100 (Supplementary Fig. 3A), indicating that $M$. hyorhinis-induced TNTs are very vulnerable to fixation and permeabilization. Thus, M. hyorhinis-induced TNTs were always observed only in live cells in further experiments.
Because DAPI (4',6-diamidino-2-phenylindole) is permeable to cell membranes and has been used as a gold standard to detect intracellular mycoplasmas (18), we stained $M$. hyorhinis-infected NIH3T3 cells with DAPI. For DAPI staining of nucleus, cells are usually fixed and permeabilized and stained with DAPI at a very low concentration (300 nM) (Supplementary Fig. 3B). But the nuclei are hardly stained even at high concentrations $(3 \mu \mathrm{M})$ in live cells after 10 minutes. In contrast, infected mycoplasma in cells was stained with $3 \mu \mathrm{M}$ DAPI for 10 min (Fig. 1C). In the P70 immunofluorescence after permeabilization (Supplementary Fig. 3B), the DAPI-stained small dots in the cytoplasm and near the plasma membrane were precisely co-localized with P70, even in the presence of gentamicin but not in the presence of MRA, indicating that DAPI could be useful for detecting intracellular M. hyorhinis. DAPI-stained dots were detected in TNTs and the cytoplasm of live NIH3T3 cells after $M$. hyorhinis infection with gentamicin but not with MRA (Fig. 1C). These data indicate that DAPI staining is useful for detecting intracellular mycoplasma in live mammalian cells.

\section{Mycoplasma transfers between NIH3T3 cells via TNTs}

Because TNTs possess $M$. hyorhinis, TNTs might be necessary for the intercellular transfer of $M$. hyorhinis between mammalian cells. To test this possibility, EGFP-expressing $\mathrm{NIH} 3 \mathrm{~T} 3$ cells were infected with $M$. hyorhinis and used as mycoplasma donor cells, while tdTomato-expressing Chang

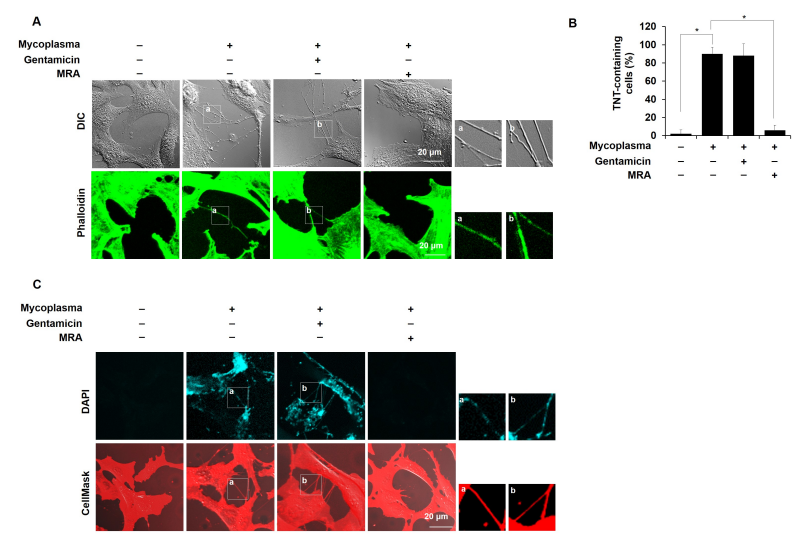

Fig. 1. $M$. hyorhinis infection induces TNT generation in NIH3T3 fibroblasts. (A) M. hyorhinis-infected NIH3T3 cells were grown in the presence of gentamicin $(600 \mathrm{ng} / \mathrm{ml})$ or Mycoplasma Removal Agent (MRA, $0.1 \mu \mathrm{g} / \mathrm{ml})$. TNTs were observed in the live state by DIC microscopy. M. hyorhinis-inducing TNTs were also observed by confocal microscopy after phalloidin-alexa fluor 488 staining in NIH3T3 cells. Boxed regions are enlarged in the bottom panels. (B) The percentage of TNT-containing cells among all cells was statistically determined and is represented as the mean \pm s.d. $* P$ $<0.01$. (C) M. hyorhinis-infected NIH3T3 cells were treated with gentamicin or MRA and stained with DAPI $(3 \mu \mathrm{M})$ and CellMast $^{\text {TM }}(5 \mu \mathrm{g} / \mathrm{ml})$ in the live state. Boxed regions are enlarged in the right panels. 
Liver cells were used as recipient cells. In the spatially separated co-culture system, the donor cells and recipient cells were cultured on the top and bottom plates and shared the same media (Fig. 2A, left panel). In the mixed co-culture system, the donor cells and recipient cells were co-cultured on the same plate to establish direct cell-to-cell contact between the donor and recipient cells (Fig. 2A, right panel). Intracellular M. hyorhinis was monitored by DAPI staining in live cells. The tdTomato-expressing recipient cells in the mixed co-culture, but not the spatially separated co-culture, contained DAPIstained mycoplasmas (Fig. 2B). In the mixed co-culture, DAPI-stained mycoplasmas were observed in TNTs between donor and recipient cells, indicating that $M$. hyorhinis disseminated from the donor cells to the recipient cells through TNTs (Fig. 2C). The transfer of intracellular $M$. hyorhinis was also monitored by P70 immunoblotting. After the spatially separated co-culture or mixed co-culture, both the EGFP-expressing donor cells and tdTomato-expressing recipient cells were sorted by a fluorescence activated cell sorter (FACS) and analyzed by P70 immunoblotting. Fig. 2D shows that the tdTomato-recipient cells in the mixed cell co-culture, but not the spatially separated co-culture, possessed P70 antigen. These data indicate that $M$. hyorhinis-induced TNTs are required for the cell-to-cell

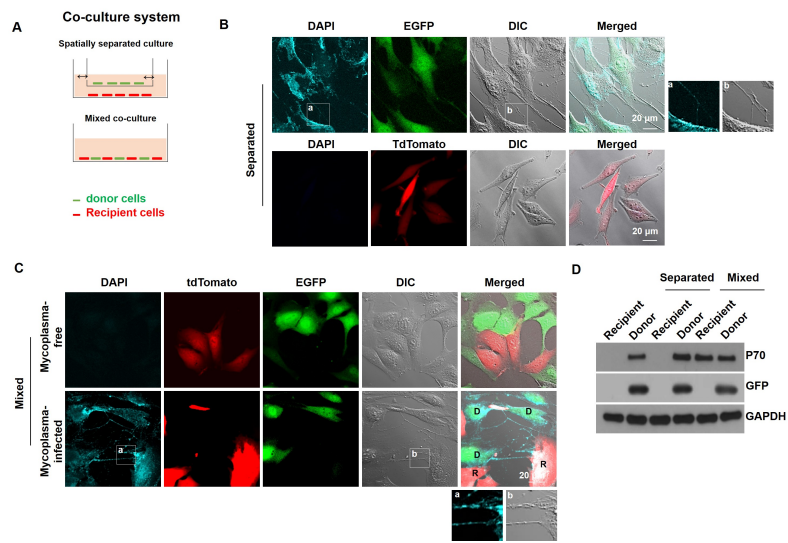

Fig. 2. Intracellular $M$. hyorhinis disseminates to surrounding cells via TNTs. (A) EGFP-expressing NIH3T3 cells were infected with $M$. hyorhinis and used as mycoplasma donor cells, and tdTomato-expressing Chang Liver cells were used as mycoplasma recipient cells. Donor and recipient cells were co-cultured in a spatially separated co-culture system or a mixed co-culture system. (B) In the spatially separated co-culture system, the donor and recipient cells were co-cultured for $24 \mathrm{~h}$ and stained with DAPI (3 $\mu \mathrm{M})$ in the live state. (C) In the mixed co-culture system, the donor and recipient cells were co-cultured for $24 \mathrm{~h}$ and stained with DAPI $(3 \mu \mathrm{M})$ in the live state. Boxed regions are enlarged in the bottom panel. $D$ and $R$ represent the donor and recipient cells, respectively. (D) After the co-culture of the donor and recipient cells in both co-culture systems, the donor and recipient cells were sorted by fluorescence-activated cell sorting (FACS) and immunoblotted for P70, GFP and GAPDH. dissemination of mycoplasmas.

\section{M. hyorhinis infection induces TNT formation by Rac1 activation}

Because mammalian TNTs are actin- and/or tubulin-based membrane protrusions, we monitored the TNTs after treating M. hyorhinis-infected NIH3T3 cells with cytochalasin B (an actin depolymerizer) or paclitaxel (a tubulin depolymerizer). The $M$. hyorhinis-induced TNTs disappeared after the treatment with cytochalasin B and paclitaxel (Fig. 3A), indicating that $M$. hyorhinis-induced TNTs are composed of actin and tubulin cytoskeletons. Because small GTP binding proteins, such as Ras, Cdc42, Rac1 and RhoA, are involved in actin remodeling, we investigated which small GTP binding proteins are involved in M. hyorhinis-induced TNT formation. The $M$. hyorhinis infection increased the activity of Rac1 and decreased the activity of Ras and Cdc42 without changing the

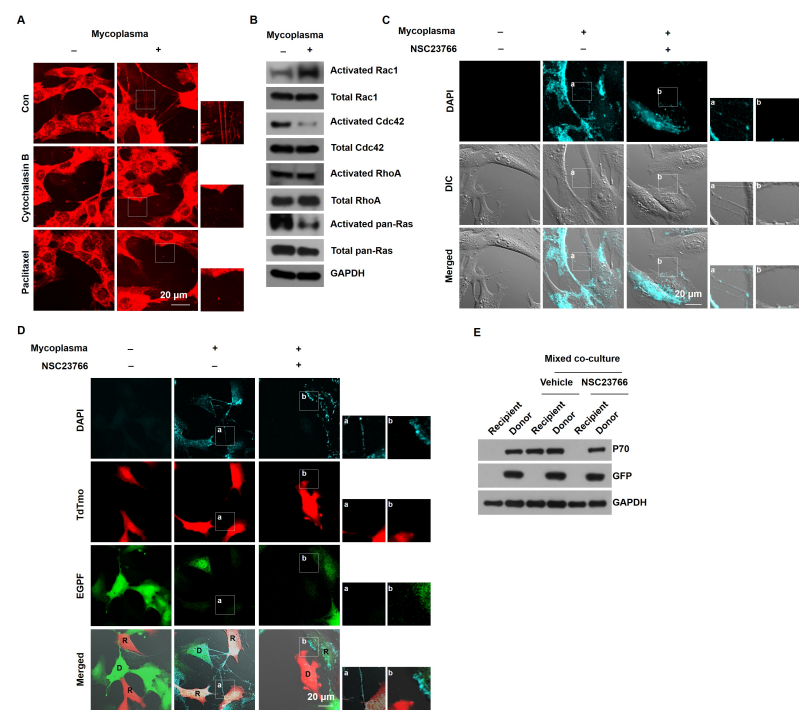

Fig. 3. Pharmacological inhibition of Rac1 prevented the cell-to-cell dissemination of $M$. hyorhinis via TNTs. (A) $M$. hyorhinis-infected NIH3T3 cells were treated with cytochalasin B $(1 \mu \mathrm{M})$ or paclitaxel $(1 \mu \mathrm{M})$ for $24 \mathrm{~h}$ and stained with CellMast ${ }^{\mathrm{TM}}$ in the live state. Boxed regions are enlarged in the right panels. (B) The activities of Rac1, Cdc42, RhoA and Ras were measured in $M$. hyorhinis-free and -infected NIH3T3 cells as described in Materials and Methods. (C) M. hyorhinis-infected NIH3T3 cells were treated with gentamicin $(600 \mathrm{ng} / \mathrm{ml})$, further incubated with the Rac1 inhibitor NSC23766 $(50 \mu \mathrm{M})$ for $24 \mathrm{~h}$, and stained with DAPI $(3 \mu \mathrm{M})$ in the live state. Boxed regions are enlarged in the right panels. (D) M. hyorhinis-infected EGFP-expressing donor NIH3T3 cells were co-cultured with tdTomato-expressing recipient Chang Liver cells in a mixed co-culture system containing NSC23766 $(50 \mu \mathrm{M})$ for $24 \mathrm{~h}$. The cells were stained with DAPI $(3 \mu \mathrm{M})$ in the live state. Boxed regions are enlarged in the right panels. (E) After the co-culture of the donor and recipient cells in a mixed co-culture system containing NSC23766 $(50 \mu \mathrm{M})$ for 24 $h$, the donor and recipient cells were sorted by FACS and immunoblotted for P70, GFP and GAPDH. 
activity of RhoA (Fig. 3B). Thus, we determined the effect of Rac1 activation on M. hyorhinis-induced TNT formation by treating $M$. hyorhinis-infected $\mathrm{NIH} 3 \mathrm{~T} 3$ cells with the Rac1-specific inhibitor NSC23766. The M. hyorhinis-induced TNTs disappeared after the treatment with NSC23766 (Fig. 3C). To investigate whether NSC23766 affects the cell-to-cell dissemination of $M$. hyorhinis via TNTs, M. hyorhinis-infected donor EGFP cells were co-cultured with recipient tdTomato cells in the presence of NSC23766. In this mixed co-culture, NSC23766 decreased DAPI-stained $M$. hyorhinis in the recipient tdTomato cells (Fig. 3D). In addition, the P70 immunoblotting showed that the NSC23766 treatment inhibited the presence of $\mathrm{P} 70$ antigen in the recipient tdTomato cells in the mixed co-culture system (Fig. 3E). These data suggest that Rac1 activation is necessary for the $M$. hyorhinis-induced formation of TNTs and cell-to-cell dissemination through TNTs.

\section{Genetic inhibition of Rac1 prevented the cell-to-cell dissemination of $M$. hyorhinis via TNTs}

To investigate whether Rac1 activation is required for $M$. hyorhinis-induced TNT formation, TNTs were observed in $M$. hyorhinis-infected NIH3T3 cells after overexpressing EGFP-Rac1, EGFP-Rac1-Q61L (constitutively active form of Rac1, EGFPCA-Rac1) and EGFP-Rac1-T17N (dominant negative form of Rac1, EGFP-DN-Rac1). The M. hyorhinis infection induced the localization of EGFP-Rac1 in TNTs in the EGFP-Rac1overexpressing NIH3T3 cells (Fig. 4A). The EGFP-CA-Rac1 overexpression induced TNT formation even without $M$. hyorhinis infection, whereas the EGFP-DN-Rac1 overexpression prevented M. hyorhinis-induced TNT formation (Fig. 4A). These results indicate that Rac1 activation is essential for $M$. hyorhinis infection-induced TNT formation. Subsequently, we examined the effect of Rac1 activation on the cell-to-cell dissemination of mycoplasmas. The $M$. hyorhinis-infected donor cells were overexpressed using EGFP-Rac1, EGFPCA-Rac1 or EGFP-DN-Rac1 and co-cultured with recipient tdTomato cells in a mixed cell culture system. DAPI-stained mycoplasma was found in the recipient tdTomato cells after the co-culture with the EGFP-Rac1 or EGFP-CA-Rac1 donor cells but not with the EGFP-DN-Rac1 donor cells (Fig. 4B, C).

To further confirm that Rac1 is required for TNT formation, we monitored $M$. hyorhinis infection-induced TNT formation after Rac1 knockdown in NIH3T3 cells. Fig. 4D and E show that $M$. hyorhinis infection-induced TNT formation was inhibited by the Rac1 knockdown. Fig. 4F also shows that DAPI-stained dots were not observed in the recipient tdTomato cells co-cultured with the Rac1-knockdown donor EGFP cells in the mixed co-culture system (Fig. 4G), suggesting that the cell-to-cell dissemination of $M$. hyorhinis from donor cells to recipient cells was prevented by the Rac1 knockdown in the donor cells. These data indicate that Rac1 is required for M. hyorhinis-induced TNT formation and cell-to cell dissemination of M. hyorhinis.
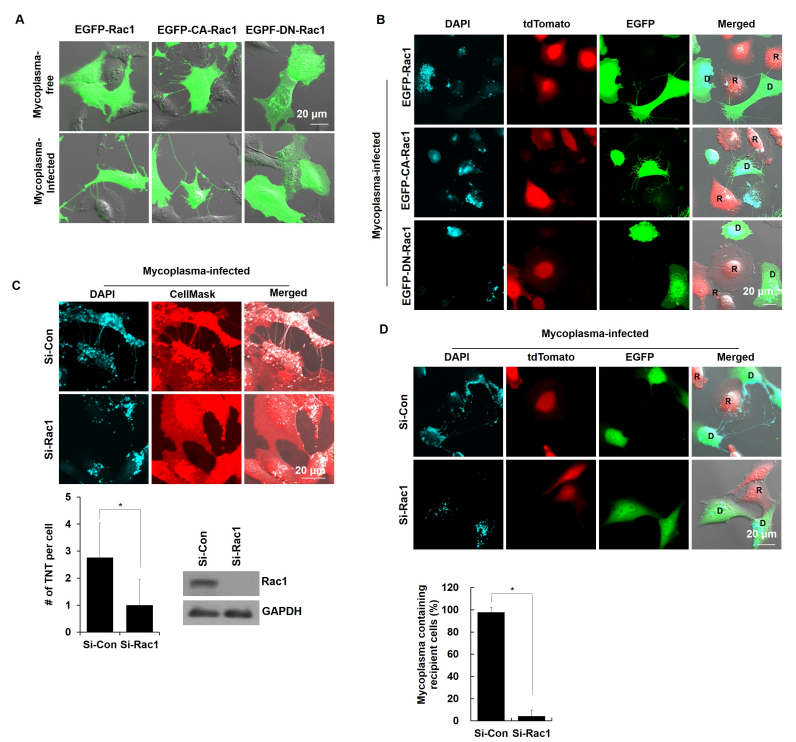

Fig. 4. Genetic inhibition of Rac1 prevented the cell-to-cell dissemination of M. hyorhinis via TNTs. (A) Mycoplasma-free and -infected NIH3T3 cells were overexpressed with an EGFP-Rac1, EGFP-Rac1-Q61L (constitutively active form of Rac1, EGFP-CA-Rac1), and EGFP-Rac1-T17N (dominant negative form of Rac1, EGFPDN-Rac1) and their TNTs were observed in the live state. Boxed regions are enlarged in the right panels. (B) M. hyorhinis-free or -infected NIH3T3 cells were overexpressed with EGFP-Rac1, EGFP-CA-Rac1 and EGFP-DN-Rac1 and co-cultured with tdTomatoexpressing Chang Liver cells in a mixed co-culture system. After $24 \mathrm{~h}$, the cells were stained with DAPI $(3 \mu \mathrm{M})$ in the live state. $\mathrm{D}$ and $\mathrm{R}$ represent the donor and recipient cells, respectively. $(\mathrm{C})$ M. hyorhinis-infected NIH3T3 cells were transfected with scrambled siRNA (si-Con) or si-Rac1 for $24 \mathrm{~h}$ and stained with DAPI $(3 \mu \mathrm{M})$ and CellMask ${ }^{T M}(5 \mu \mathrm{g} / \mathrm{ml})$ in the live state. Boxed regions are enlarged in the bottom panels. The number of TNTs per cell was counted and is represented as the mean \pm s.d. ${ }^{*} \mathrm{P}$ $<0.01$. The expression level of Rac1 and GAPDH was determined by immunoblotting. (D) M. hyorhinis-infected EGFP-expressing NIH3T3 cells (donor cells) were transfected with scrambled si-Con or si-Rac1 for $24 \mathrm{~h}$ and co-cultured with tdTomato-expressing cells (recipient cells) in a mixed co-culture system. After $24 \mathrm{~h}$ of co-culture, the cells were stained with DAPI $(3 \mu \mathrm{M})$ in the live state. The percentage of DAPI-stained cells among all recipient cells was statistically determined and is represented as the mean \pm s.d. ${ }^{*} P<0.01$. One hundred cells were observed in ten different fields under each condition.

\section{DISCUSSION}

The dissemination of pathogens, such as viruses and bacteria, to surrounding host cells without leaving the host cells represents a powerful way of avoiding exposure to the host's immune system. Our data provide a novel mechanism of the cell-to-cell dissemination of $M$. hyorhinis through TNTs. The intracellular invasion of mycoplasma triggers Rac1 activation, leading to TNT formation in various mammalian cells. The TNTs were utilized as a novel intercellular pathway for the 
cell-to-cell dissemination of mycoplasma. Thus, the cell-to-cell dissemination of mycoplasma through TNTs can potentially overcome anti-bacterial innate immune defenses and the action of many antibiotics. Our data demonstrate that intracellular mycoplasmas survived in the presence of the antibiotic gentamicin and disseminated to surrounding host cells via TNTs.

Actin remodeling for TNT formation is regulated by the activation of small GTP binding proteins, such as RalA, Cdc42, RhoA and Rac1 (22). For example, Cdc42 might be necessary for TNT formation because its knockdown and dominant negative form dramatically reduce the number of TNTs in HIV-1-infected dendritic cells and Fas-stimulated T lymphocytes $(23,24)$. In contrast, the Cdc42/IRSp53/VASP network inhibited TNT production in neurons (25). We also excluded the role of Cdc42 in $M$. hyorhinis infection-induced TNT formation because the $M$. hyorhinis infection decreased the activity of Cdc42 (Fig. 3A). Instead of Cdc42, we focused on the role of Rac1 activation in M. hyorhinis infection-induced TNT formation because the activity of Rac1 was increased in M. hyorhinis-infected fibroblasts, and its pharmacological, knockdown and dominant negative form prevented $M$. hyorhinis infection-induced TNT formation (Fig. 3 and Fig. 4). TNT formation is known to be induced by an actin-driven protrusion mechanism in which protrusions formed in one or both cells are connected or fused with the cell membrane of another cells (7). The Rho family of GTPases such as Rac1 and CDC42 are involved in actin polymerization of these protrusions (22). Mycoplasma-induced TNT had an actin-based structure similar to other pathogen-induced TNTs (Fig. 1A and Fig. 3A). The blocking of TNT formation by knockdown and dominant negative form of Rac1 ultimately results from the inhibition of actin polymerization in the protrusion. In RAW/LR5 macrophages, the Rac1-Arp2/3-WASP pathway is also required for TNT formation (26). Several pathogens activate small GTP binding proteins in host cells to facilitate their proliferation. The gram-negative bacteria Shigella induces Cdc42 activation to increase bacterial invasion, and the Salmonella IpgB1 protein functions as a RhoA GTP binding protein to induce actin remodeling in host cells $(27,28)$.

Mycoplasma is resistant to beta-lactam antibiotics (e.g., penicillin) because it lacks a cell wall; thus, macrolide, tetracycline, or fluoroquinolone classes have been used to treat mycoplasma-induced diseases, such as M. pneumoniaeinduced pneumonia. However, the emergence of macrolideresistant mycoplasma strains requires new treatments for mycoplasma-induced diseases (29). Our data show that $M$. hyorhinis exploits TNTs for cell-to-cell dissemination. Although extracellular $M$. hyorhinis was removed by gentamicin in the infected cells, intracellular mycoplasmas were disseminated to surrounding cells via TNTs. Because the cell-to-cell dissemination of $M$. hyorhinis was prevented by the pharmacological or genetic inhibition of Rac1 (Fig. 3 and Fig. 4), Rac1 inhibition may be a novel strategy to alleviate mycoplasma-induced diseases.

\section{MATERIALS AND METHODS}

See Supplementary information.

\section{ACKNOWLEDGEMENTS}

This work was supported by the National Research Foundation of Korea (NRF) grant funded by the Korea government (MSIT) (NRF-2015R1A5A1009024) and supported by Basic Science Research Program through the National Research Foundation of Korea (NRF) funded by the Ministry of Education (NRF-2017R1D1A1B03030413).

\section{CONFLICTS OF INTEREST}

The authors have no conflicting interests.

\section{REFERENCES}

1. Stappenbeck TS and Miyoshi H (2009) The role of stromal stem cells in tissue regeneration and wound repair. Science 324, 1666-1669

2. Tkach $M$ and Thery C (2016) Communication by extracellular vesicles: Where we are and where we need to go. Cell 164, 1226-1232

3. Schiller PC, D'Ippolito G, Brambilla R, Roos BA and Howard GA (2001) Inhibition of gap-junctional communication induces the trans-differentiation of osteoblasts to an adipocytic phenotype in vitro. J Biol Chem 276, 14133-14138

4. Ramirez-Weber FA and Kornberg TB (1999) Cytonemes: cellular processes that project to the principal signaling center in Drosophila imaginal discs. Cell 97, 599-607

5. Gerdes HH, Rustom A and Wang X (2013) Tunneling nanotubes, an emerging intercellular communication route in development. Mech Dev 130, 381-387

6. Rainy N, Chetrit D, Rouger V et al (2013) H-Ras transfers from $B$ to $T$ cells via tunneling nanotubes. Cell Death Dis 4, e726

7. Rustom A, Saffrich R, Markovic I, Walther P and Gerdes $\mathrm{HH}$ (2004) Nanotubular highways for intercellular organelle transport. Science 303, 1007-1010

8. Hurtig J, Chiu DT and Onfelt B (2010) Intercellular nanotubes: insights from imaging studies and beyond. Wiley Interdiscip Rev Nanomed Nanobiotechnol 2, 260-276

9. Abounit S, Bousset L, Loria F et al (2016) Tunneling nanotubes spread fibrillar alpha-synuclein by intercellular trafficking of lysosomes. EMBO J 35, 2120-2138

10. Gousset K, Schiff E, Langevin C et al (2009) Prions hijack tunnelling nanotubes for intercellular spread. Nat Cell Biol 11, 328-336

11. Tardivel M, Begard S, Bousset $L$ et al (2016) Tunneling nanotube (TNT)-mediated neuron-to neuron transfer of pathological Tau protein assemblies. Acta Neuropathol 
Commun 4, 117

12. Kumar A, Kim JH, Ranjan $P$ et al (2017) Influenza virus exploits tunneling nanotubes for cell-to-cell spread. Sci Rep 7, 40360

13. Sherer NM, Lehmann MJ, Jimenez-Soto LF, Horensavitz C, Pypaert M and Mothes W (2007) Retroviruses can establish filopodial bridges for efficient cell-to-cell transmission. Nat Cell Biol 9, 310-315

14. Panasiuk M, Rychlowski M, Derewonko $N$ and BienkowskaSzewczyk K (2018) Tunneling nanotubes as a novel route of cell-to-cell spread of herpesviruses. J Virol 92, e0009018

15. Okafo G, Prevedel L and Eugenin E (2017) Tunneling nanotubes (TNT) mediate long-range gap junctional communication: Implications for HIV cell to cell spread. Sci Rep 7, 16660

16. Onfelt B, Nedvetzki S, Benninger RK et al (2006) Structurally distinct membrane nanotubes between human macrophages support long-distance vesicular traffic or surfing of bacteria. J Immunol 177, 8476-8483

17. Drexler HG and Uphoff CC (2002) Mycoplasma contamination of cell cultures: Incidence, sources, effects, detection, elimination, prevention. Cytotechnology 39, 75-90

18. Rottem S (2003) Interaction of mycoplasmas with host cells. Physiol Rev 83, 417-432

19. Borovsky Z, Tarshis M, Zhang P and Rottem S (1998) Protein kinase $C$ activation and vacuolation in HeLa cells invaded by Mycoplasma penetrans. J Med Microbiol 47, 915-922

20. Winner F, Rosengarten R and Citti C (2000) In vitro cell invasion of Mycoplasma gallisepticum. Infect Immun 68, 4238-4244
21. Yavlovich A, Tarshis M and Rottem S (2004) Internalization and intracellular survival of Mycoplasma pneumoniae by non-phagocytic cells. FEMS Microbiol Lett 233, 241-246

22. Kimura S, Hase K and Ohno H (2013) The molecular basis of induction and formation of tunneling nanotubes. Cell Tissue Res 352, 67-76

23. Arkwright PD, Luchetti F, Tour J et al (2010) Fas stimulation of $\mathrm{T}$ lymphocytes promotes rapid intercellular exchange of death signals via membrane nanotubes. Cell Res 20, 72-88

24. Nikolic DS, Lehmann M, Felts R et al (2011) HIV-1 activates Cdc42 and induces membrane extensions in immature dendritic cells to facilitate cell-to-cell virus propagation. Blood 118, 4841-4852

25. Delage E, Cervantes DC, Penard E et al (2016) Differential identity of Filopodia and Tunneling Nanotubes revealed by the opposite functions of actin regulatory complexes. Sci Rep 6, 39632

26. Hanna SJ, McCoy-Simandle K, Miskolci V et al (2017) The Role of Rho-GTPases and actin polymerization during Macrophage Tunneling Nanotube Biogenesis. Sci Rep 7, 8547

27. Alto NM, Shao F, Lazar CS et al (2006) Identification of a bacterial type III effector family with $G$ protein mimicry functions. Cell 124, 133-145

28. Tran Van Nhieu G, Caron E, Hall A and Sansonetti PJ (1999) IpaC induces actin polymerization and filopodia formation during Shigella entry into epithelial cells. EMBO J 18, 3249-3262

29. Cardinale F, Chironna M, Chinellato I, Principi N and Esposito S (2013) Clinical relevance of Mycoplasma pneumoniae macrolide resistance in children. J Clin Microbiol 51, 723-724 